\title{
First Report on Multidrug-Resistant Methicillin-Resistant Staphylococcus aureus Isolates in Children Admitted to Tertiary Hospitals in Vietnam ${ }^{\mathbb{S}}$
}

\author{
Nguyen Thai Son ${ }^{1}, \mathrm{Vu}$ Thi Thu Huong${ }^{2}, \mathrm{Vu}$ Thi Kim Lien ${ }^{2}$, Do Thi Quynh Nga', Tran Thi Hai Au ${ }^{2}$, \\ Tang Thi Nga ${ }^{2}$, Le Nguyen Minh $\mathrm{Hoa}^{3}$, and Tran Quang Binh ${ }^{2,4 *}$ \\ ${ }^{1}$ Vietnam Military Medical University, Hanoi 151000, Vietnam \\ ${ }^{2}$ National Institute of Hygiene and Epidemiology, Hanoi 100000, Vietnam \\ ${ }^{3}$ National Hospital for Tropical Diseases, Hanoi 100000, Vietnam \\ ${ }^{4}$ Dinh Tien Hoang Institute of Medicine, Hanoi 155300, Vietnam
}

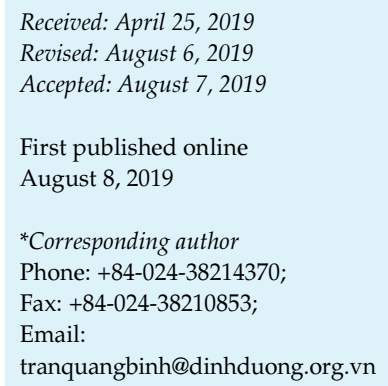

S upplementary data for this paper are available on-line only at http://jmb.or.kr.

pISSN 1017-7825, eISSN 1738-8872

\section{Copyright(C) 2019 by}

The Korean Society for Microbiology and Biotechnology
The extensive distribution of multidrug-resistant (MDR) methicillin-resistant Staphylococcus aureus (MRSA) poses a threat to healthcare worldwide. This study aimed to investigate the MDR and molecular patterns of MRSA isolates in children admitted to the two biggest tertiary care pediatric hospitals in northern and southern Vietnam. A total of 168 MRSA strains were collected to determine antibiotic susceptibility by minimum inhibitory concentration tests. Antibiotic-resistant genes, pulsed-field gel electrophoresis, staphylococcal cassette chromosome mec (SCCmec) typing, and multilocus sequence typing were used for the molecular characterization of MRSA. Among the total strains, the MDR rate $(51.8 \%)$ was significantly higher in the northern hospital than in the southern hospital $(73 \%$ vs. 39\%, $p<$ 0.0001). The MDR-MRSA with the highest rates were "ciprofloxacin-erythromycin-gentamicintetracyclines" (35.6\%), followed by "erythromycin-tetracycline-chloramphenicol" $(24.1 \%)$, and "ciprofloxacin-erythromycin-gentamicin" (19.5\%), showing an accumulative total of 79.3\%. The most susceptible antibiotics were rifampicin (100\%) and vancomycin (100\%), followed by doxycycline $(94.0 \%)$, meropenem $(78.0 \%)$, and cefotaxime $(75.0 \%)$. The SCCmecII strains showed greater resistance to gentamicin, ciprofloxacin, tetracycline, meropenem and cephalosporins compared with the other strains. The SCCmecII strains exhibited the highest rate in the tested genes (aacA/aphD: $55.2 \%$, ermA/B/C: $89.7 \%$, and tetK/M: $82.8 \%$ ). ST5SCCmecII was the predominant clone in the northern hospital, whereas SCCmecIVa was more pronounced in the southern hospital. In conclusion, our results raised concerns about the predominant MDR-MRSA strains in the pediatric hospitals in Vietnam. The north-south difference in the antibiotic resistance patterns and genetic structure of MRSA suggests different MRSA origins and various uses of antimicrobial agents between the two regions.

Keywords: Methicillin-resistant, multidrug-resistant, Staphylococcus aureus, molecular pattern, children, Vietnam

\section{Introduction}

Staphylococcus aureus is a common bacterium that can cause a variety of diseases from localized to systemic infections [1]. Methicillin-resistant S. aureus (MRSA) is a major cause of nosocomial infections worldwide and is becoming increasingly prevalent in community settings [2,
3]. The emergence and spread of multidrug-resistant (MDR) MRSA pose a serious problem in the treatment and control of staphylococcal infections, thereby threatening global human health [4, 5]. In northern Vietnam, the nasopharyngeal carriage of $S$. aureus is observed in onethird of the Vietnamese population [6], and the population structure of MRSA differs from that in other Asian 
countries $[7,8]$. In southern Vietnam, the MRSA rates were $74.1 \%$ in hospitals and $30.1 \%$ in communities in 2004-2006 [9]. Most of the abovementioned studies were conducted on adult samples. An outbreak of severe communityacquired MRSA infections was reported in nine children in Ho Chi Minh City [10]. However, information about MDR and the molecular epidemiology of MRSA infections in Vietnamese children remains scarce.

The antimicrobial susceptibility patterns of $S$. aureus were identified using minimum inhibitory concentrations (MICs), and the presence of genes conferring resistance to S. aureus was detected by multiplex PCR. The different types of staphylococcal cassette chromosome mec (SCCmec) were identified to check for the distribution of SCCmec types among MRSA isolates. Multilocus sequence typing (MLST) was used to determine the sequence type (ST) to understand the geographical spread of MRSA isolates. Pulsed-field gel electrophoresis (PFGE) was used to assess the genetic similarity among MRSA isolates [11, 12]. In this study, we applied the above methods to investigate MDR and characterize MRSA isolates in children admitted to the biggest tertiary care pediatric hospitals in northern and southern Vietnam.

\section{Materials and Methods}

\section{Ethics Statement}

Isolates were obtained from patients with verbal consent from their parents as part of the standard care for patients in pediatric hospitals. Isolates were coded to protect the patients' identities and stored for treatment and study purposes. This study protocol was reviewed and approved by the Ethical Review Board (IRB No. 18IRB) of the National Institute of Hygiene and Epidemiology, Hanoi, Vietnam.

\section{Bacterial Isolates and Clinical Information}

A total of 168 non-duplicate clinical strains of MRSA were collected retrospectively between December 2011 and May 2014 in the two biggest pediatric hospitals located in two distinct geographic regions in Vietnam: 63 strains were obtained from the National Hospital of Pediatrics in Hanoi in northern Vietnam, and 105 strains were obtained from the Children's Hospital No. 2 in Ho Chi Minh City in southern Vietnam (Table S1). The two hospitals were tertiary care general hospitals with 1,400-2,000 beds. The strains were previously isolated from blood samples $(\mathrm{N}=77)$, other body fluids $(\mathrm{N}=9)$, respiratory samples $(\mathrm{N}=25)$, and skin lesions $(\mathrm{N}=57)$. The median age (interquartile range) of children was 9.6 (1.3-26.8) months. Isolates were identified as S. aureus using Gram staining, positive catalase test, coagulase test, and a commercial latex agglutination kit (Pastorex Staph Plus, Bio-Rad, France). S. aureus isolates were further confirmed by ID-GP Card in VITEK 2 Compact (BioMérieux) in the two hospitals. MRSA isolates were identified using the MIC breakpoint of cefoxitin in accordance with the criteria of Clinical and Laboratory Standards Institute [13] and confirmed by the presence of the mecA533 or mecA310 genes using multiplex PCR $[14,15]$. Clinical data, including general demographic information, laboratory results, and treatment, were collected from medical records. MRSA infections that occurred either more than $48 \mathrm{~h}$ after hospital admission or with healthcare risk factors were classified as healthcare-associated (HA) MRSA infection as reported previously, and those that occurred in inpatients within $48 \mathrm{~h}$ of admission without healthcare risk factors were classified as community-associated (CA) MRSA infections [16]. If an S. aureus infection did not meet these criteria, then it was considered a CA infection.

\section{Antimicrobial Susceptibility Tests}

Antibiotic susceptibility testing of all the bacterial strains was performed using microdilution and agar dilution methods in accordance with the M100-S26 Guidelines of the Clinical and Laboratory Standards Institute [13]. The 12 tested antibiotics were cefuroxime (CFR), cefotaxime (CFT), cefepime (CFP), meropenem (MER), gentamicin (GEN), ciprofloxacin (CIP), erythromycin (ERY), rifampicin (RIF), tetracycline (TET), doxycycline (DOX), chloramphenicol (CHL), and vancomycin (VAN). The MICs of the tested antimicrobials were measured by agar dilution. MIC 90 and MIC50 were defined as the lowest concentrations of the antibiotic at which $90 \%$ and $50 \%$ of the isolates were inhibited, respectively.

On the basis of the standardized international terminology to describe acquired resistance profiles in 2012 [17], all the MRSA isolates in the present study were classified as MDR. No isolate was considered extensively drug-resistant. By contrast, many previous studies used the MDR definition for MRSA (MDRMRSA) such that MRSA isolates were classified as MDR if they were non-susceptible to three or more different classes without beta-lactams $[4,9,18,19]$. To compare our findings with those of previous studies, we used the same previous MDR-MRSA definition with non-beta-lactam antimicrobial types, including fluoroquinolones (CIP), macrolides (ERY), aminoglycosides (GEN), TETs (TET and/or DOX), phenicols (CHL), ansamycins (RIF), and glycopeptides (VAN) [18]. S. aureus ATCC 29213 was used as a quality control strain in the MIC experiments.

\section{Analysis of Antibiotic-Resistant Genes}

All the $S$. aureus strains were screened for the presence of the mecA gene with $533 \mathrm{bp}$ PCR product (mecA533) as previously described [14]. Thirty-four mecA533-negative isolates were screened for the presence of the mecA310 gene using another PCR method [15]. Genes conferring resistance to other antibiotics of TET (tetK and tet $\mathrm{M})$, aminoglycosides (aac $\mathrm{A} / a p h \mathrm{D})$, and macrolides and lincosamide (erm $\mathrm{A}$, erm $\mathrm{B}$, and erm $\mathrm{C}$ ) were detected by multiplex PCR assays as previously reported [20]. 


\section{Molecular Characterization of the MRSA Strains}

All the MRSA isolates from the two hospitals were selected for SCCmec typing. SCCmec types were determined by multiplex PCR as previously reported [12]. SCCmec type IV was further tested by the subtyping method [21]. SCCmec types that could not be assigned to any known type by the above methods were classified as non-typeable.

PFGE was performed using 30 units of SmaI enzyme per sample [22] for selected MRSA strains that were classified either as MDR or identified as causes of sepsis, shock, or death. The PFGE patterns of the MRSA isolates were determined by cluster analysis using BioNumerics software version 6.6 (Applied-Maths, SintMartens-Latem, Belgium). Pairwise similarities were calculated using the Dice coefficient (optimization, 1\%; band tolerance, $1.5 \%)$. The unweighted-pair group method using average algorithm was used to generate a dendrogram. Isolates with $\geq 80 \%$ similarity were assigned in a PFGE cluster. Clusters that included $\geq 10$ isolates were considered major lineages. Sixteen strains were randomly selected in all clusters for MLST as described elsewhere. [11]. The allelic profiles and STs were assigned by the MLST website (http:/ / saureus.mlst.net).

\section{Statistical Analysis}

Categorical variables were expressed in numbers and proportions (\%). The chi-square test or Fisher's exact test was used to compare the distribution of the categorical variables. All statistical procedures were performed using Stata software version 11.0. A $p$ value less than 0.05 was considered statistically significant.

\section{Results}

In the total sample count, the highest antibiotic resistance

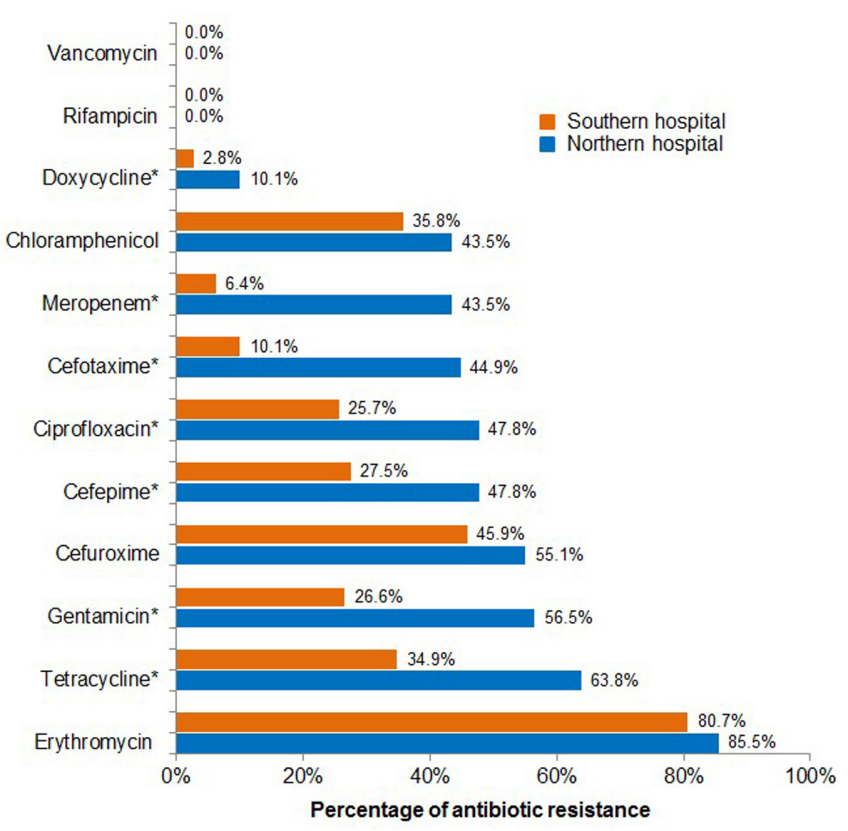

Fig. 1. Comparison of antibiotic resistance methicillin-resistant Staphylococcus aureus isolates between the two hospitals.

*Significant difference between northern and southern hospitals at $p<0.05$.

frequency was found in ERY (81.5\%). Antibiotic resistance rates of one-third to half of the tested isolates were observed in CFR (52.4\%), TET (45.2\%), GEN (38.1\%), CFP (37.5\%), CHL (36.3\%), and CIP (35.7). The most susceptible antibiotics were RIF (100\%) and VAN (100\%), followed by

Table 1. Antimicrobial resistance profiles by HA-MRSA and CA-MRSA in two pediatric hospitals, Vietnam.

\begin{tabular}{|c|c|c|c|c|c|c|c|}
\hline \multirow{2}{*}{$\begin{array}{c}\text { Antimicrobial } \\
\text { category }\end{array}$} & \multirow{2}{*}{$\begin{array}{l}\text { Antimicrobial } \\
\text { agent }\end{array}$} & \multicolumn{2}{|l|}{ HA-MRSA } & \multirow[t]{2}{*}{$p$-value } & \multicolumn{2}{|l|}{ CA-MRSA } & \multirow[t]{2}{*}{$p$-value } \\
\hline & & $\begin{array}{l}\text { Northern hospital } \\
\qquad(\mathrm{N}=48)\end{array}$ & $\begin{array}{l}\text { Southern hospital } \\
\qquad(\mathrm{N}=82)\end{array}$ & & $\begin{array}{l}\text { Northern hospital } \\
\qquad(\mathrm{N}=15)\end{array}$ & $\begin{array}{l}\text { Southern hospital } \\
\qquad(\mathrm{N}=23)\end{array}$ & \\
\hline Ansamycins & Rifampicin & $0(0)$ & $0(0)$ & - & $0(0)$ & $0(0)$ & - \\
\hline Fluoroquinolones & Ciprofloxacin & $28(58.3)$ & $24(29.3)$ & 0.001 & $4(26.7)$ & $4(17.4)$ & $0.687^{*}$ \\
\hline Macrolides & Erythromycin & $40(83.3)$ & $67(81.7)$ & 0.815 & $13(86.7)$ & $17(73.9)$ & $0.440^{*}$ \\
\hline Phenicols & Chloramphenicol & $14(29.2)$ & $29(35.4)$ & 0.468 & $10(66.7)$ & $8(34.8)$ & 0.054 \\
\hline \multirow[t]{2}{*}{ Tetracyclines } & Tetracycline & $30(62.5)$ & $26(31.7)$ & 0.001 & $10(66.7)$ & $10(43.5)$ & 0.162 \\
\hline & Doxycycline & $5(10.4)$ & $2(2.4)$ & $0.100^{*}$ & $2(13.3)$ & $1(4.3)$ & $0.550^{*}$ \\
\hline Carbapenems & Meropenem & $26(54.2)$ & $5(6.1)$ & $<0.0001$ & $4(26.7)$ & $2(8.7)$ & $0.188^{*}$ \\
\hline \multirow{2}{*}{ Cephalosporins } & Cefotaxime & $27(56.2)$ & $9(11.0)$ & $<0.0001$ & $4(26.7)$ & $2(8.7)$ & $0.188^{*}$ \\
\hline & Cefepime & $29(60.4)$ & $25(30.5)$ & 0.001 & $4(26.7)$ & $5(21.7)$ & $1.000^{*}$ \\
\hline
\end{tabular}

*Fisher's exact test. CA, community-associated; HA, hospital-associated; MRSA, methicillin-resistant S. aureus. 
Table 2. Antimicrobial resistance profiles by SCCmec types of methicillin-resistant Staphylococcus aureus isolates in two pediatric hospitals, Vietnam.

\begin{tabular}{|c|c|c|c|c|c|c|}
\hline $\begin{array}{l}\text { Antimicrobial } \\
\text { category }\end{array}$ & $\begin{array}{l}\text { Antimicrobial } \\
\text { agent }\end{array}$ & $\begin{array}{c}\text { SCCmecII } \\
(\mathrm{N}=29)\end{array}$ & $\begin{array}{l}\text { SCCmecIII } \\
(\mathrm{N}=64)\end{array}$ & $\begin{array}{c}\text { SCCmecIV } \\
\left(\mathrm{N}=66^{*}\right)\end{array}$ & $\begin{array}{l}\text { Others } \\
(\mathrm{N}=9)\end{array}$ & $p$-value \\
\hline Aminoglycosides & Gentamicin & $28(96.6)$ & $9(14.1)$ & $23(34.8)$ & $4(44.4)$ & $<0.0001$ \\
\hline Ansamycins & Rifampicin & $0(0)$ & $0(0)$ & $0(0)$ & $0(0)$ & \\
\hline Fluoroquinolones & Ciprofloxacin & $25(82.6)$ & $8(12.5)$ & $23(34.8)$ & $4(44.4)$ & $<0.0001$ \\
\hline Glycopeptides & Vancomycin & $0(0)$ & $0(0)$ & $0(0)$ & $0(0)$ & \\
\hline Macrolides & Erythromycin & $28(96.6)$ & $61(95.3)$ & $39(59.1)$ & $9(100)$ & $<0.0001$ \\
\hline Phenicols & Chloramphenicol & $5(17.2)$ & $39(60.9)$ & $12(18.2)$ & $5(55.6)$ & $<0.0001$ \\
\hline \multirow[t]{2}{*}{ Tetracyclines } & Tetracycline & $23(79.3)$ & $41(64.1)$ & $11(16.7)$ & $1(11.1)$ & $<0.0001$ \\
\hline & Doxycycline & $5(17.2)$ & $3(4.7)$ & $0(0)$ & $2(22.2)$ & 0.001 \\
\hline Carbapenems & Meropenem & $25(86.2)$ & $8(12.5)$ & $1(1.5)$ & $3(33.3)$ & $<0.0001$ \\
\hline \multirow[t]{3}{*}{ Cephalosporins } & Cefuroxime & $27(93.1)$ & $16(25.0)$ & $40(60.6)$ & $5(55.6)$ & $<0.0001$ \\
\hline & Cefotaxime & $25(86.2)$ & $10(15.6)$ & $6(9.1)$ & $1(11.1)$ & $<0.0001$ \\
\hline & Cefepime & 26 (89.7) & $13(20.3)$ & $21(31.8)$ & $3(33.3)$ & $<0.0001$ \\
\hline
\end{tabular}

*66 SCCmecIV: 59 SCCmecIVa, 6 SCCmecIVc/IVe, 1 SCCmecIVg. Others: Non-typeable SCCmec and SCCmecI.

DOX (94\%), MER (78.0\%), and CFT (75.0\%). A comparison of the antibiotic resistance of MRSA isolates between the two hospitals is presented in Fig. 1. The MRSA isolates exhibited significantly higher antibiotic resistance in the northern hospital than in the southern hospital to DOX, MER, CFT, CIP, CFP, GEN, and TET ( $p<0.05)$.

Table 1 shows the antimicrobial resistance profiles of HA-MRSA and CA-MRSA in the two pediatric hospitals. With regard to HA-MRSA, a significant north-south difference of antimicrobial agents in the two regions was found in GEN (62.5\% vs. $29.3 \%$ ), CIP (58.3\% vs. $29.3 \%$ ), TET (62.5\% vs. $31.7 \%$ ), MER (54.2\% vs. $6.1 \%)$, CFT (56.2\% vs. $11 \%)$, and CFP (60.4\% vs. $30.5 \%)$. The antimicrobial resistance profiles of CA-MRSA isolates in the two pediatric hospitals in all antibiotics tested were not significantly different.

The distribution of SCCmec types of MRSA isolates in the northern and southern hospitals is shown in Table S2. The majority of the MRSA isolates belonged to SCCmecIV $(39.3 \%)$, followed by SCCmecIII $(38.1 \%)$ and SCCmecII $(17.3 \%)$ in the total samples. The north-south difference in the SCCmec types was observed in the present study: SCCmecII (41.3\%), SCCmecIII (33.3\%), and SCCmecIV (22.2\%) were found in northern Vietnam, whereas SCCmecII (2.9\%), SCCmecIII $(41.0 \%)$, and SCCmecIV (49.5\%) were observed in southern Vietnam $(p<0.0001)$.

Table 2 shows the antimicrobial resistance profiles by SCCmec types of MRSA isolates in the two pediatric hospitals. Overall, the SCCmecII strains showed greater resistance to GEN, CIP, TET, MER, and cephalosporins

Table 3. Distribution of antibiotic-resistant genes by SCCmec types of methicillin-resistant Staphylococcus aureus strains.

\begin{tabular}{|c|c|c|c|c|c|c|}
\hline Gene & SCCmecII & SCCmecIII & SCCmecIV & Others & Total & $p$-value \\
\hline mecA & $29(100)$ & $64(100)$ & $66(100)$ & $9(100)$ & $168(100)$ & 1.00 \\
\hline$a a c \mathrm{~A} / a p h \mathrm{D}$ & $16(55.2)$ & $6(9.4)$ & $24(36.4)$ & $3(36.4)$ & $49(29.2)$ & $<0.0001$ \\
\hline ermA & $24(82.8)$ & $4(6.2)$ & $0(0)$ & 1 (11.1) & 29 (17.3) & $<0.0001$ \\
\hline erm $\mathrm{B}$ & $4(13.8)$ & $45(70.3)$ & $29(43.9)$ & $6(56.7)$ & $84(50.0)$ & $<0.0001$ \\
\hline erm C & $0(0)$ & $0(0)$ & $12(18.2)$ & $2(22.2)$ & $14(8.3)$ & $<0.0001$ \\
\hline tetK & $1(3.4)$ & $43(67.2)$ & $10(15.2)$ & $2(22.2)$ & $56(33.3)$ & $<0.0001$ \\
\hline tet $\mathrm{M}$ & $24(82.8)$ & $3(4.7)$ & $1(1.5)$ & $0(0)$ & $28(16.7)$ & $<0.0001$ \\
\hline tet $\mathrm{K} / \mathrm{M}$ & $24(82.8)$ & $44(68.8)$ & $11(16.7)$ & $2(22.2)$ & $81(42.8)$ & $<0.0001$ \\
\hline
\end{tabular}

Data are number (\%). Others: Non-typeable SCCmec and SCCmecI. 
(CFR, CFT, and CFP) than the other strains. The SCCmecII strains also had the highest rate in the tested genes (aacA/ aphD: 55.2\%, ermA/B/C: $89.7 \%$, tetK/M: 82.8\%; Table 3). A statistically significant difference was observed in the distribution of the tested genes (except for mecA) among the SCCmec types $(p<0.0001)$.

The MIC values by the SCCmec types of MRSA isolates from the children in the two pediatric hospitals are shown in Table S3. The SCCmecII isolates showed the highest MIC50 and MIC90 for almost all antibiotics tested among the SCCmec types. Increased MIC50 levels were observed in ERY $(>128 \mu \mathrm{g} / \mathrm{ml})$, CHL $(8 \mu \mathrm{g} / \mathrm{ml})$, CFR $(32 \mu \mathrm{g} / \mathrm{ml})$, CFT $(8 \mu \mathrm{g} / \mathrm{ml})$, and CFP $(16 \mu \mathrm{g} / \mathrm{ml})$. MIC90 $\geq 128 \mu \mathrm{g} / \mathrm{ml}$ was noted in GEN, ERY, CHL, CFR, CFT, and CFP.

The MDR-MRSA rates significantly decreased with age: $77.1 \%$ in neonates, $54.7 \%$ in infants, and $36.2 \%$ in pediatrics $(p<0.0001)$. Table 4 shows the MDR patterns by the SCCmec types of the MDR-MRSA isolates in the northern and southern hospitals in Vietnam. The rate of the MDRMRSA isolates was 51.8\% (87/168). The MDR-MRSA isolates had the highest co-resistance rate to four antibiotic classes "CIP-ERY-GEN-TETs" (31/87, 35.6\%), followed by "ERY-TET-CHL" (21/87, 24.1\%) and "CIP-ERY-GEN" (17/ $87,19.5 \%$ ), with an accumulative total of $79.3 \%$. Among 87 MDR-MRSA strains, the frequencies of the isolates that showed co-resistance to five, four, and three classes of antibiotics without beta-lactams were 1 (1.1\%), 40 (46\%), and $46(52.9 \%)$, respectively. In addition, significantly higher MDR-MRSA isolates were found in the northern hospital than in the southern hospital (73 vs. 39\%, $p<$

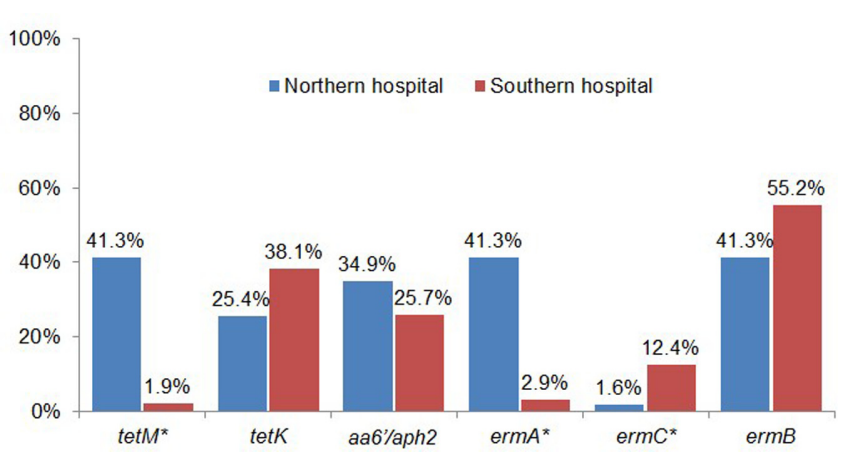

Fig. 2. Distribution of genes conferring antibiotic resistance among methicillin-resistant Staphylococcus aureus isolates in the northern and southern hospitals, Vietnam.

*Significant difference between northern and southern hospitals at $p<0.05$.

0.0001). The northern hospital exhibited a higher rate of coresistance to four antibiotics "CIP-ERY-GEN-TETs" (SCCmecII: $43.5 \%$ in northern hospital vs. $4.9 \%$ in southern hospital) and a lower rate of three co-resistance agents "CIP-ERY-GEN" (SCCmecIVa: 2.2\% in northern hospital vs. $26.8 \%$ in southern hospital) in comparison with the southern hospital.

Fig. 2 shows the distribution of genes conferring antibiotic resistance among the MRSA isolates in the northern and southern hospitals in Vietnam. A significant difference was found between the two regions in the distribution of tet $\mathrm{M}$, erm $\mathrm{A}$, and erm $\mathrm{C}(p<0.01)$. Among 168 MRSA isolates, the most predominant genes conferring

Table 4. Multidrug-resistant patterns by SCCmec types of methicillin-resistant Staphylococcus aureus isolates in two pediatric hospitals, Vietnam.

\begin{tabular}{|c|c|c|c|c|}
\hline $\begin{array}{c}\text { No. of } \\
\text { co-resistance agent }\end{array}$ & $\begin{array}{c}\text { Antimicrobial resistance } \\
\text { phenotype }\end{array}$ & $\begin{array}{l}\text { Total } \\
(\mathrm{N}=87)\end{array}$ & $\begin{array}{l}\text { Northern hospital } \\
\qquad(\mathrm{N}=46)\end{array}$ & $\begin{array}{l}\text { Southern hospital } \\
\qquad(\mathrm{N}=41)\end{array}$ \\
\hline 5 & CIP-ERY-GEN-TET-CHL & $\mathrm{II}(1)$ & $\mathrm{II}(1)$ & 0 \\
\hline \multirow[t]{3}{*}{4} & CIP-ERY-GEN-TETs & $\mathrm{II}(22), \mathrm{III}(3), \mathrm{IVa}(5), \mathrm{NA}(1)$ & $\mathrm{II}(20)^{*}, \mathrm{III}(3)$ & II(2), IVa(5), NA(1) \\
\hline & CIP-ERY-GEN-CHL & $\mathrm{II}(1), \mathrm{IVa}(2)$ & $\mathrm{II}(1)$ & $\operatorname{IVa}(2)$ \\
\hline & ERY-GEN-TET-CHL & $\mathrm{I}(1), \mathrm{III}(3), \mathrm{IVa}(1), \operatorname{IVg}(1)$ & I(1), III(1), IVa(1) & $\mathrm{III}(2), \operatorname{IVg}(1)$ \\
\hline \multirow[t]{7}{*}{3} & ERY-TET-CHL & III(20), IVc/VIe(1) & III(10); IVc/VIe(1) & III(10) \\
\hline & CIP-ERY-GEN & $\mathrm{II}(1), \mathrm{III}(2), \mathrm{IVa}(12), \mathrm{NA}(2)$ & $\mathrm{II}(1), \mathrm{III}(1), \mathrm{IVa}(1), \mathrm{NA}(1)$ & $\mathrm{III}(1), \operatorname{IVa}(11)^{*}, \mathrm{NA}(1)$ \\
\hline & CIP-ERY-TET & $\mathrm{III}(1), \operatorname{IVa}(1)$ & $\operatorname{III}(1)$ & $\operatorname{IVa}(1)$ \\
\hline & CIP-ERY-CHL & $\operatorname{IVa}(1), \mathrm{NA}(1)$ & 0 & $\operatorname{IVa}(1), \mathrm{NA}(1)$ \\
\hline & GEN-CHL-DOX & $\mathrm{II}(1)$ & $\mathrm{II}(1)$ & 0 \\
\hline & ERY-GEN-CHL & $\mathrm{II}(1), \mathrm{III}(1)$ & 0 & $\mathrm{II}(1), \mathrm{III}(1)$ \\
\hline & ERY-GEN-TET & IVa (1) & IVa (1) & 0 \\
\hline
\end{tabular}

CHL: Chloramphenicol; CIP: ciprofloxacin; ERY: erythromycin; GEN: gentamicin; TETs (TET: tetracycline and/or DOX: doxycycline). Roman numerals indicate SCCmec types. Data in parentheses are number of isolates. *Statistically significant difference between Northern and Southern hospitals at $p<0.01$. 


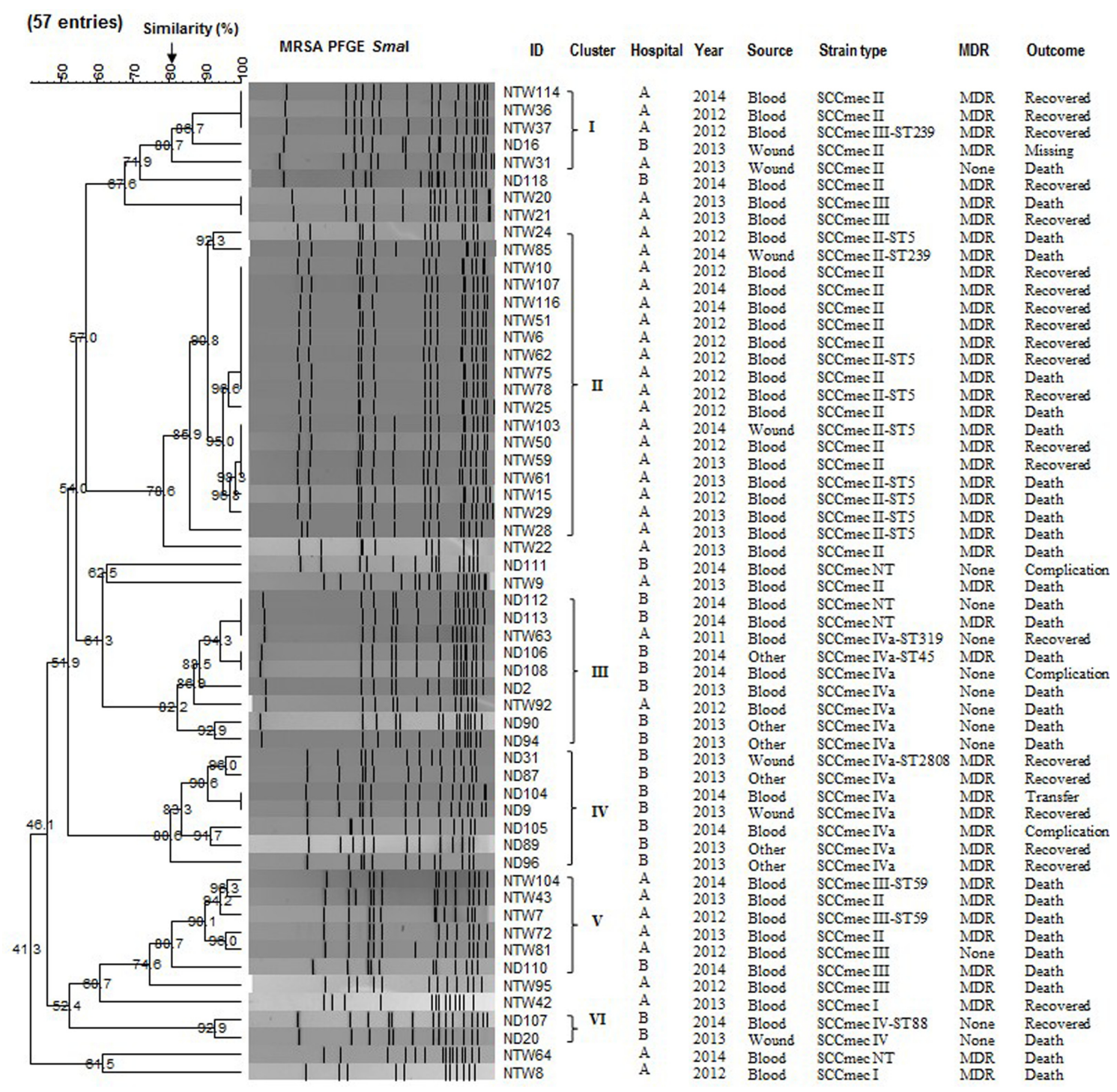

Fig. 3. Dendrogram based on PFGE patterns of 57 multidrug-resistant and/or death-associated MRSA isolates in the pediatric hospitals, Vietnam.

Numbers along the branches indicate percentage of similarity among isolates. The isolates with the similarity over $80 \%$ were clustered from I to VI. MRSA: methicilin-resistant Staphylococcus aureus; PFGE: pulsed-field gel electrophoresis; A: National Hospital of Pediatrics; B: Children's Hospital No. 2; MDR: multidrug-resistant.

antibiotic resistance were as follows: $\mathrm{ermA} / \mathrm{B} / \mathrm{C}(72.6 \%)$, tet $\mathrm{K} / \mathrm{M}(42.8 \%)$, and $a a c \mathrm{~A} / \mathrm{aph} \mathrm{D}(29.2 \%)$, which corresponded to the antibiotic resistance phenotypes ERY (81.5\%), TET $(46.4 \%)$, and GEN $(38.1 \%)$, respectively.

PFGE analysis of the MDR and/or death-associated isolates recovered in this study revealed six PFGE clusters (Fig. 3). Cluster II was the largest and the only major lineage that contained 18 MDR-MRSA isolates from the National Institute of Pediatrics in northern Vietnam. All of the 18 isolates in cluster II were SCCmecII. Two STs (ST5 and ST239) were identified by MLST among 9 out of 18 isolates in the major cluster II, in which ST5-SCCmecII was the predominant profile (8 isolates). Sporadic STs were observed in minor clusters: ST239-SCCmecIII in cluster I, ST59-SCCmecIII in cluster V, ST88-SCCmecIV in cluster VI, SCCmecIVa with ST45 or ST319 in cluster III, and ST2808SCCmecIVa in cluster IV. Clusters III and IV contained 14 (out of 16) MDR-MRSA isolates from Children's Hospital No. 2 in southern Vietnam between 2012 and 2014. SCCmecIVa was predominant in clusters III and IV (14/16). 


\section{Discussion}

S. aureus silently exists as a commensal bacterium that colonizes human mucosal surfaces, but it can also threaten our life as an opportunistic, conditionally pathogenic microorganism. MRSA is responsible for several infections that are difficult to treat in humans. The first MRSA was found among S.aureus clinical isolates in 1961 [23], less than 1 year after the introduction of methicillin into clinical practice; however, MRSA was likely acquired in the mid1940s [24]. In the Vietnamese population, the first MRSA report in 2004-2006 [9] showed that MRSA rates were $30.1 \%$ in communities and $74.1 \%$ in hospitals in southern Vietnam. These rates were higher than those in general Asians: MRSA accounted for $25.5 \%$ of CA S. aureus infections and $67.4 \%$ of $\mathrm{HA}$ infections. In Asian populations, the MDR rates were $73.1 \%$ and $83.7 \%$ in CA-MRSA and HAMRSA isolates, respectively $(p=0.001)$, but no such data were reported in Vietnam [9]. Several subsequent studies did not report MDR-MRSA isolates [6-8, 10, 25]. The present study is the first report of MDR-MRSA isolates in Vietnamese children. Our results raise concerns about the predominant MDR-MRSA isolates in pediatric hospitals in Vietnam. The MDR rate was $51.8 \%$ in MRSA isolates; it was significantly higher in the northern hospital compared with that in the southern hospital (73 vs. 39\%, $p<0.0001$ ), possibly reflecting differences in the use of antimicrobial agents between the two regions. The MDR rate in MRSA isolates in this study was lower than that in other countries. It varied in nosocomial children worldwide: $100 \%$ in China [19], versus $66.7 \%$ in Chicago, USA [18]. The HA-MRSA isolates from children and adults exhibited similar non- $\beta$ lactam antimicrobial drug resistance rates in Chicago, USA [18].

One of the major findings of the present study is the detailed MIC profile by the SCCmec types of MRSA isolates in 12 commonly used antibiotics in Vietnamese pediatric hospitals. Among the SCCmec types, SCCmecII showed the highest MIC50 and MIC90 for almost all antibiotics tested. Increased MIC50 and MIC90 levels were observed in ERY (MIC50>128 $\mu \mathrm{g} / \mathrm{ml}$ ), CHL, and cephalosporins (CFR, CFT, and CFP). Fortunately, RIF (100\% sensitive, MIC range: $0.06-2 \mu \mathrm{g} / \mathrm{ml})$ and VAN (100\% sensitive, MIC range: $0.5-$ $2 \mu \mathrm{g} / \mathrm{ml}$ ) were fully susceptible antibiotics to MRSA isolates, and DOX was susceptible in most cases (94\%) in the Vietnamese pediatric hospitals. VAN has been considered the standard therapy for serious MRSA infections for a long time. The decreased susceptibility of S. aureus to VAN has been reported since 1997 [26]. Our study showed that VAN was fully sensitive to MRSA, which was consistent with previous reports from many Asian countries (Vietnam, Hong Kong, Philippines, Sri Lanka, Korea, China, Thailand, Nepal, Taiwan, and India) $[8,9,22,25,27,28]$, whereas its high doses have been recommended to maintain efficacy in other countries [29]. VAN-sensitive MRSA was reported in eight pediatric hospitals in mainland China [19], whereas reduced VAN susceptibility was found in MRSA and methicillin-sensitive $S$. aureus clinical strains in two adult hospitals in northeast China [30]. A national antimicrobial resistance monitoring system for $S$. aureus must be maintained.

In terms of the SCCmec types in MRSA isolates, a previous study on adult subjects in southern Vietnam reported [9] that the most frequent SCCmec type is SCCmecIII (CA: 51.6\%, HA: 47.6\%), followed by SCCmecII (CA: $15.6 \%$, HA: 33.3\%), SCCmecI (CA: 12.9\%, HA: 4.8\%), and SCCmecIV (CA: 3.2\%, HA: 0\%). The present study on pediatric subjects in southern Vietnam reported a different trend: SCCmecIV (CA: 34.8\%, HA: 53.7\%), SCCmecIII (CA: 47.8\%, HA: 39\%), and SCCmecII (CA: 13\%, HA: 0\%). Only one study analyzed the SCCmec types in children: an outbreak of CA-MRSA [10] reported ST59-SCCmecV (N=5), ST59-SCCmecIVa $(\mathrm{N}=1)$, and one allele variant of ST45 $(\mathrm{N}=1)$ in nine children. Notably, the north-south difference of the SCCmec types was observed in the present study: SCCmecII $(40.6 \%)$ was predominant in northern Vietnam, whereas SCCmecIV (47.3\%) was more pronounced in southern Vietnam $(p<0.0001)$, showing the different genetic structures of MRSA between the two regions. Indeed, a significant difference was observed between the two regions in the distribution of genes $(\operatorname{tet} \mathrm{M}, \operatorname{erm} \mathrm{A}$, and erm $\mathrm{C})$ conferring antibiotic resistance $(p<0.01)$.

The observed differences in the antimicrobial susceptibilities and genotypes between northern and southern MRSA isolates may be explained by the following factors. (i) Farmers from the north and south have different knowledge and practices on antibiotic use (many antibiotics used to treat human diseases are used in agriculture) [31]. (ii) Antibiotic prescription in private clinics is not based on antibiotic susceptibility tests, and private clinics are abundant in the Red River Delta (58\%) compared with that the Mekong River Delta (27\%) [32]. (iii) The prevalence of inappropriate indications for antibiotic prescriptions is high in hospitals in Vietnam [33], and most antibiotics are sold without a prescription in pharmacies: $88 \%$ and $91 \%$ in urban and rural areas, respectively [34].

Among 16 MDR-MRSA isolates from the southern pediatric hospital in Ho Chi Minh City, SCCmecIVa was 
predominant $(14 / 16)$ in clusters III and IV, indicating that SCCmecIVa could be expanded clonally in the hospital and may result in intra-hospital patient-to-patient spread. SCCmec type IVa isolates are known to be more prevalent in South Korea than in other Asian countries [35]. The strain NN47 was isolated in 2008 from a Japanese girl and described as the first USA300 transmitted among people in Japan [36]. The USA300 clone, that is, ST8-MRSA-IVa, has become the prevalent CA-MRSA strain in the United States since it was first reported in 2000 as a cause of skin and soft tissue infections. It is also recognized as an emerging nosocomial MRSA [37]. SCCmecIVa was reported to be predominant in a major hospital in Lebanon [38] and in a community environment in Australia [39]. Given that the USA300 clone is highly transmissible and virulent, surveillance in Vietnam is of utmost importance.

MLST STs, namely, CA-MRSA-ST59, CA-MRSA-ST239, and HA-MRSA-ST239, were previously reported in southern Vietnam [9,40]. In northern Vietnam, HA-MRSA: ST59 (15/25), ST45 (5/25), ST239 (2/25), ST188 (2/25), and ST834 (1/25); and CA-MRSA: ST45 (11/28), ST59 (8/28), ST188 $(1 / 28)$, ST25 $(1 / 28), \operatorname{ST} 88(1 / 28), \operatorname{ST} 121(2 / 28)$, ST1232 (1/28), ST15 (1/28), ST406 (1/28), and ST942 $(1 / 28)$ were described [7]. Previous studies reported the predominant STs of ST59 and ST45 in the north and ST59 and ST239 in the south for both HA-MRSA and CA-MRSA isolates from adults. In 2006, an outbreak of severe CAMRSA pediatric infections of ST59 occurred in southern Vietnam following routine vaccination injection [10]. ST45 and ST239 were also found in this study. The present study indicated the predominant clone of ST5-SCCmecII. It initially recognized ST239-SCCmecII and ST319-SCCmecVIa in the northern hospital and ST2808-SCCmecIVa in the southern hospital.

In Asian countries, ST239-SCCmecIII and ST5-SCCmecII have been reported as the two major endemic MRSA clones prevalent in hospitals with a unique geographic distribution and evolutionary MRSA clone patterns. Almost all MRSA strains from Korea and Japan belong to ST5-SCCmecII (named as the New York/Japan MRSA clone), whereas most MRSA isolates from other Asian countries belong to ST239-SCCmecIII [40]. After 15 years, these HA-MRSA clones possibly spread to communities in countries including Korea, Taiwan, Thailand, Vietnam, and Sri Lanka [9]. The major clonal lineages of HA-MRSA isolates were ST239-MRSA-SCCmecIII in Thailand, Korea, Vietnam, Taiwan, and India and ST5-MRSA-SCCmectype II in Taiwan, Philippines, Hong Kong, Sri Lanka, and Korea. ST5-SCCmecII (USA100) has not been previously reported in Vietnam. In the present study, ST5-SCCmecII was initially recognized as the predominant clone in the northern hospital, suggesting the intra-hospital transmission of this clone.

The ST239-MRSA clone was first discovered in Brazil [41], and it has since become widely spread in various hospitals. ST239-SCCmecIII is commonly found in HAMRSA in Asian populations [9,40,42]. Interestingly, ST239SCCmecII was first reported in this study, in line with a report in a Malaysian hospital [43]. This ST239-SCCmecII clone is more frequent $(8.1 \%, 16 / 198)$ in HA-MRSA isolates in Chinese hospitals than in other Asian countries [44]. Thus, evaluations of the geographic distribution and evolutionary pattern of this MRSA clone are crucial.

The present study has some limitations. First, as a retrospective study, the database collected from medical records was subject to missing data, and the incidence rates of $S$. aureus infection outbreak and non-MDR-MRSA infections could not be identified. Second, MLST and spa and arg typing methods could not be performed in all samples although the ST types were identified in all clusters. Third, the sample size of CA-MRSA was relatively small, so significant differences in antibiotic resistance of CA-MRSA between the two regions could not be detected. Finally, our work included only some genes conferring antibiotic resistance. A comprehensive image of the genetic patterns of MRSA isolates in Vietnam may be necessary in the future.

In conclusion, the present study highlighted concerns about the predominant MDR-MRSA isolates from pediatric patients in Vietnam. The most effective antibiotics to treat S. aureus infections in children were found to be VAN and RIF. These findings support a different characteristic of the molecular structure and antibiotic resistance of MRSA isolates from pediatric hospitals between northern and southern Vietnam.

\section{Acknowledgments}

We acknowledge the health staff and colleagues in the National Institute of Pediatrics and Children's Hospital No. 2 for their cooperation and kind support in providing S. aureus isolates. We would like to thank Dr. Panida Nobthai and Dr. Oralak Serichantalergs (Armed Forces Research Institute of Medical Sciences, Bangkok, Thailand) for their skillful technical assistance and data analysis.

This study was supported by the National Foundation for Science and Technology Development, Vietnam (Grant No. 106.06-2012.25). The funder had no role in the study 
design, data collection and analysis, decision to publish, or preparation of the manuscript.

\section{Conflict of Interest}

The authors have no financial conflicts of interest to declare.

\section{References}

1. Wertheim HF, Melles DC, Vos MC, van Leeuwen W, van Belkum A, Verbrugh HA, et al. 2005. The role of nasal carriage in Staphylococcus aureus infections. Lancet Infect. Dis. 5: 751-762.

2. Boucher HW, Corey GR. 2008. Epidemiology of methicillinresistant Staphylococcus aureus. Clin. Infect. Dis. 46: S344-S349.

3. David MZ, Daum RS. 2010. Community-associated methicillinresistant Staphylococcus aureus: epidemiology and clinical consequences of an emerging epidemic. Clin. Microbiol. Rev. 23: 616-687.

4. Lee AS, de Lencastre H, Garau J, Kluytmans J, MalhotraKumar S, Peschel A, et al. 2018. Methicillin-resistant Staphylococcus aureus. Nat. Rev. Dis. Primers. 4: 18033.

5. Sola C, Paganini H, Egea AL, Moyano AJ, Garnero A, Kevric I, et al. 2012. Study group of CA-MRSA in children, Argentina-2007, Lopardo H, Bocco JL. Spread of epidemic MRSA-ST5-IV clone encoding PVL as a major cause of community onset staphylococcal infections in Argentinean children. PLoS One. 7: e30487.

6. Van Nguyen K, Zhang T, Thi Vu BN, Dao TT, Tran TK, Thi Nguyen DN, et al. 2014. Staphylococcus aureus nasopharyngeal carriage in rural and urban northern Vietnam. Trans. R. Soc. Trop. Med. Hyg. 108: 783-790.

7. Ngoc Thi Vu B, J Jafari A, Aardema M, Kieu Thi Tran H, Ngoc Thi Nguyen D, Tuyet Dao T, et al. 2016. Population structure of colonizing and invasive Staphylococcus aureus strains in northern Vietnam. J. Med. Microbiol. 65: 298-305.

8. Dat VQ, Vu HN, Nguyen The H, Nguyen HT, Hoang LB, $\mathrm{Vu}$ Tien Viet $\mathrm{D}$, et al. 2017. Bacterial bloodstream infections in a tertiary infectious diseases hospital in Northern Vietnam: aetiology, drug resistance, and treatment outcome. BMC Infect. Dis. 17: 493.

9. Song JH, Hsueh PR, Chung DR, Ko KS, Kang CI, Peck KR, et al. 2011. Spread of methicillin-resistant Staphylococcus aureus between the community and the hospitals in Asian countries: an ANSORP study. J. Antimicrob. Chemother. 66: 1061-1069.

10. Tang CT, Nguyen DT, Ngo TH, Nguyen TM, Le VT, To SD, et al. 2007. An outbreak of severe infections with communityacquired MRSA carrying the Panton-Valentine leukocidin following vaccination. PLoS One 2: e822.
11. Enright MC, Day NP, Davies CE, Peacock SJ, Spratt BG. 2000. Multilocus sequence typing for characterization of methicillin-resistant and methicillin-susceptible clones of Staphylococcus aureus. J. Clin. Microbiol. 38: 1008-1015.

12. Oliveira DC, de Lencastre H. 2002. Multiplex PCR strategy for rapid identification of structural types and variants of the mec element in methicillin-resistant Staphylococcus aureus. Antimicrob. Agents. Chemother. 46: 2155-2161.

13. Clinical and Laboratory Standards Institute (2016) Performance standards for antimicrobial susceptibility testing; 25th informational supplement. CLSI document M100-S26. Clinical and Laboratory Standards Institute, Wayne, PA.

14. Maes N, Magdalena J, Rottiers S, De Gheldre Y, Struelens MJ. 2002. Evaluation of a triplex PCR assay to discriminate Staphylococcus aureus from coagulase-negative Staphylococci and determine methicillin resistance from blood cultures. J. Clin. Microbiol. 40: 1514-1517.

15. McClure JA, Conly JM, Lau V, Elsayed S, Louie T, Hutchins W, et al. 2006. Novel multiplex PCR assay for detection of the staphylococcal virulence marker Panton-Valentine leukocidin genes and simultaneous discrimination of methicillinsusceptible from -resistant staphylococci. J. Clin. Microbiol. 44: 1141-1144.

16. Klevens RM, Morrison MA, Fridkin SK, Reingold A, Petit S, Gershman K, et al. 2006. Community-associated methicillinresistant Staphylococcus aureus and healthcare risk factors. Emerg. Infect. Dis. 12: 1991-1993.

17. Magiorakos A-P, Srinivasan A, Carey RB, Carmeli Y, Falagas ME, Giske CG, et al. 2012. Multidrug-resistant, extensively drug-resistant and pandrug-resistant bacteria: an international expert proposal for interim standard definitions for acquired resistance. Clin. Microbiol. Infect. 18: 268-281.

18. David MZ, Crawford SE, Boyle-Vavra S, Hostetler MA, Kim DC, Daum RS. 2006. Contrasting pediatric and adult methicillin-resistant Staphylococcus aureus isolates. Emerg. Infect. Dis. 12: 631-637.

19. Wang L, Liu Y, Yang Y, Huang G, Wang C, Deng L, et al. 2012. Multidrug-resistant clones of community-associated meticillin-resistant Staphylococcus aureus isolated from Chinese children and the resistance genes to clindamycin and mupirocin. J. Med. Microbiol. 61: 1240-1247.

20. Strommenger B, Kettlitz C, Werner G, Witte W. 2003. Multiplex PCR assay for simultaneous detection of nine clinically relevant antibiotic resistance genes in Staphylococcus aureus. J. Clin. Microbiol. 41: 4089-4094.

21. Milheiriço C, Oliveira DC, de Lencastre H. 2007. Multiplex PCR strategy for subtyping the staphylococcal cassette chromosome mec type IV in methicillin-resistant Staphylococcus aureus: 'SCCmec IV multiplex'. J. Antimicrob. Chemother. 60: 42-48.

22. Lee TM, Yang MC, Yang TF, Lee PL, Chien HI, Hsueh JC, et al. 2015. Molecular characterization of community- and healthcare-associated methicillin resistant Staphylococcus 
aureus isolates in southern Taiwan. Microb. Drug. Resist. 21: 610-621.

23. Oliveira DC, Tomasz A, H. de Lencastre. 2002. Secrets of success of a human pathogen: molecular evolution of pandemic clones of meticillin-resistant Staphylococcus aureus. Lancet Infect. Dis. 2: 180-189.

24. Harkins CP, Pichon B, Doumith M, Parkhill J, Westh H, Tomasz A, et al. 2017. Methicillin-resistant Staphylococcus aureus emerged long before the introduction of methicillin into clinical practice. Genome Biol. 18: 130.

25. Thuy DB, Campbell J, Nhat LTH, Hoang NVM, Hao NV, Baker S, et al. 2018. Hospital-acquired colonization and infections in a Vietnamese intensive care unit. PLoS One 13: e0203600.

26. Hiramatsu K, Hanaki H, Ino T, Yabuta K, Oguri T, Tenover FC. 1997. Methicillin-resistant Staphylococcus aureus clinical strain with reduced vancomycin susceptibility. J. Antimicrob. Chemother. 40: 135-136.

27. Kshetry AO, Pant ND, Bhandari R, Khatri S, Shrestha KL, Upadhaya SK, et al. 2016. Minimum inhibitory concentration of vancomycin to methicillin resistant Staphylococcus aureus isolated from different clinical samples at a tertiary care hospital in Nepal. Antimicrob. Resist. Infect. Control. 5: 27.

28. Kaur DC, Chate SS. 2015. Study of antibiotic resistance pattern in methicillin resistant Staphylococcus aureus with special reference to newer antibiotic. J. Glob. Infect. Dis. 7: 78-84.

29. Moise PA, Amodio-Groton M, Rashid M, Lamp KC, Hoffman-Roberts HL, Sakoulas G, et al. 2013. Multicenter evaluation of the clinical outcomes of daptomycin with and without concomitant beta-lactams in patients with Staphylococcus aureus bacteremia and mild to moderate renal impairment. Antimicrob. Agents. Chemother. 57: 1192-1200.

30. Hu J, Ma XX, Tian Y, Pang L, Cui LZ, Shang H. 2013. Reduced vancomycin susceptibility found in methicillinresistant and methicillin-sensitive Staphylococcus aureus clinical isolates in Northeast China. PLoS One 8: e73300.

31. Pham DK, Chu J, Do NT, Brose F, Degand G, Delahaut P, et al. 2015. Monitoring antibiotic use and residue in freshwater aquaculture for domestic use in Vietnam. Ecohealth 12: 480489.

32. Nguyen MP, Wilson A. 2017. How Could Private Healthcare Better Contribute to Healthcare Coverage in Vietnam? Int. J. Health Policy. Manag. 6: 305-308.

33. Thu TA, Rahman M, Coffin S, Harun-Or-Rashid M, Sakamoto J, Hung NV. 2012. Antibiotic use in Vietnamese hospitals: a multicenter point-prevalence study. Am. J. Infect. Control. 40: 840-844.
34. Nga do TT, Chuc NT, Hoa NP, Hoa NQ, Nguyen NT, Loan HT, et al. 2014. Antibiotic sales in rural and urban pharmacies in northern Vietnam: an observational study. BMC Pharmacol. Toxicol. 15: 6.

35. Park C, Shin HH, Kwon EY, Choi SM, Kim SH, Park SH, et al. 2009. Two variants of staphylococcal cassette chromosome mec type IVA in community-associated meticillin-resistant Staphylococcus aureus strains in South Korea. J. Med. Microbiol. 58: 1314-1321.

36. Higuchi W, Mimura S, Kurosawa Y, Takano T, Iwao Y, Yabe S, et al. 2010. Emergence of the community-acquired methicillin-resistant Staphylococcus aureus USA300 clone in a Japanese child, demonstrating multiple divergent strains in Japan. J. Infect. Chemother. 16: 292-297.

37. Tenover FC, and Goering RV. 2009. Methicillin-resistant Staphylococcus aureus strain USA300: origin and epidemiology. J. Antimicrob. Chemother. 64: 441-446.

38. Harastani HH, Araj GF, Tokajian ST. 2014. Molecular characteristics of Staphylococcus aureus isolated from a major hospital in Lebanon. Int. J. Infect. Dis. 19: 33-38.

39. Coombs GW, Monecke S, Pearson JC, Tan HL, Chew YK, Wilson L, et al. 2011. Evolution and diversity of communityassociated methicillin-resistant Staphylococcus aureus in a geographical region. BMC Microbiol. 11: 215.

40. Ko KS, Lee JY, Suh JY, Oh WS, Peck KR, Lee NY, et al. 2005. Distribution of major genotypes among methicillin-resistant Staphylococcus aureus clones in Asian countries. J. Clin. Microbiol. 43: 421-426.

41. Teixeira LA, Resende CA, Ormonde LR, Rosenbaum R, Figueiredo AM, de Lencastre H, et al. 1995. Geographic spread of epidemic multiresistant Staphylococcus aureus clone in Brazil. J. Clin. Microbiol. 33: 2400-2404.

42. Liu Y, Wang H, Du N, Shen E, Chen H, Niu J, et al. 2009. Molecular evidence for spread of two major methicillinresistant Staphylococcus aureus clones with a unique geographic distribution in Chinese hospitals. Antimicrob. Agents. Chemother. 53: 512-518.

43. Samat Muttaqillah NA, Hussin S, Neoh HM, Noordin A, Ding $\mathrm{CH}$, Wahab AA, et al. 2015. Clonal diversity of methicillin-resistant Staphylococcus aureus in UKM Medical Centre: characterisation by multilocus sequence typing of different SCCmec type representatives. Sains Malaysiana. 44: 1315-1323.

44. Li T, Song Y, Zhu Y, Du X, Li M. 2013. Current status of Staphylococcus aureus infection in a central teaching hospital in Shanghai, China. BMC Microbiol. 13: 153. 